versity of Montreal Press, Montreal, Canada, 1969), p. 240 .

${ }^{16} \mathrm{~J}$. L. Fowler, in Proceedings of the International Conference on Properties of Nuclear States, Montreal, Canada, 1969, edited by M. Harvey et al. (University of Montreal Press, Montreal, Canada, 1969), p. 334.

${ }^{17}$ H. S. Köhler, Nucl。Phys。88, 529 (1966); L. R. B. Elton, Phys. Letters 25B, 60 (1967).

${ }^{18} \mathrm{This}$ is Elton's $-E_{R}$ (corr) modified by reducing his correction for center-of-mass energy by a factor of 2 , since only the c.m. kinetic energy and not its potential energy is contained in the BHF binding energy.

${ }^{19}$ R. L. Becker and M. R. Patterson, to be published。 ${ }^{20}$ K. H. Purser, et al., Nucl. Phys. A132, 75 (1969).

${ }^{21}$ L. R. B. Elton and S. J. Webb, to be published.
Their fit to elastic $e^{-}$scattering from $\mathrm{Ca}$ is shown in Fig. 2 of R. C. Barrett, in Proceedings of the International Conference on Properties of Nuclear States, Montreal, Canada, 1969, edited by H. Harvey et al. (University of Montreal Press, Montreal, Canada, 1969), p. 109.

${ }^{22}$ Occupation probabilities have been incorporated in matrix BHF calculations: R. L. Becker and K. T. R. Davies, in Proceedings of the International Conference on Properties of Nuclear States, Montreal, Canada, 1969, edited by M. Harvey et al. (University of Montreal, Montreal, Canada, 1969), p. 164. See also R. M. McCarthy and K. T. R. Davies, Phys. Rev. (to be published), and K. T. R. Davies and M. Baranger, to be published.

\title{
INFLUENCE OF NUCLEAR FORCES ON THE COULOMB BARRIER IN HEAVY-ION REACTIONS*
}

\author{
Hartmut Holm and Walter Greiner \\ Institut für Theoretische Physik der Universität, Frankfurt am Main, Germany \\ (Received 15 December 1969)
}

\begin{abstract}
The influence of the Coulomb and nuclear forces on the Coulomb barrier in heavy-ion reactions is studied in a dynamical classical model. It is shown that the fusion barrier is smaller than the conventional Coulomb barrier of two undeformed nuclei. The model yields a dynamical picture of the excitation mechanism of surface vibrations and giant resonances. It is suggested that-due to nuclear forces-the excitation of the octupole mode is strongly enhanced over the excitation of the quadrupole mode in experiments at the Coulomb barrier.
\end{abstract}

The long-range Coulomb interaction causes an increase of the Coulomb barrier in heavy-ion reactions by exciting vibrations, rotations, and giant resonances. This effect has been investigated previously. ${ }^{1-4}$ It is of great importance for the fusion process in the collision of heavy nuclei. We have now studied the lowering of the Coulomb barrier by nuclear forces within the same dynamical classical model.

The total Hamiltonian for central collisions of two spherical nuclei is

$$
H=\frac{p R^{2}}{2 m}+H_{\mathrm{vib}(1)}+H_{\mathrm{vib}(2)}+H_{\mathrm{dip}(1)}+H_{\mathrm{dip}(2)}+H_{\mathrm{dip} \text { quad(1) }}+H_{\mathrm{dip} \text { quad(2) }}+H_{\text {Coul int }}+H_{\text {Yukawa int }} \text {, }
$$

where $m$ is the reduced mass. The first terms are explained in detail in Holm, Scheid, and Greiner ${ }^{2}$ and Holm et al. ${ }^{4}$ : They describe, respectively, the relative kinetic energy of the ions and-within the framework of the collective model ${ }^{5,6}$ - the giant resonances $\left(H_{\text {dip }}\right)$ and the quadrupole and octupole surface vibrations $\left(H_{\text {vib }}\right)$. The vibrational amplitudes are defined by the usual expansion of the radius in spherical harmonics. In the harmonic approximation one obtains for the vibrational Hamiltonian

$$
H_{\mathrm{vlb}}=\frac{1}{2} \sum_{l=2,3}(-1)^{1}(2 l+1)^{1 / 2}\left\{\frac{1}{B_{1}}\left[\pi^{[l]} \times \pi^{[l]}\right]^{[0]}+C_{l}\left[\alpha^{[l]} \times \alpha^{[1]}\right]^{[0]}\right\},
$$

and for the giant resonances

$$
H_{\mathrm{dip}}=-\frac{1}{2} \sqrt{3}\left\{\left[1 / B_{1}\right]\left[\pi^{[1]} \times \pi^{[1]}\right]^{[0]}+C_{1}\left[d^{[1]} \times d^{[1]}\right]^{[0]}\right\} .
$$

$B_{2}, B_{3}, C_{2}$, and $C_{3}$ are taken from the transition probabilities and the excitation energies (for the data, see Wong ${ }^{7}$ and Hansen and Nathan ${ }^{8}$ ), whereas $B_{1}$ and $C_{1}$ are given by ${ }^{5} B_{1}=M^{*} Z A / V N k^{2}$ and $C_{1}=8 \kappa Z^{2} /$ $V A$. $V$ is the nuclear volume; $M^{*}=M /(1+a)$ is an effective nucleon mass adjusted to the experimental excitation energy of the giant resonances $E_{\mathrm{dip}}=80 A^{-1 / 3} \mathrm{MeV} . \quad k=2.0815 / R_{0}$ is the wave number of the giant-resonance oscillations, $R_{0}=1.2 A^{1 / 3} \mathrm{fm}$ denotes the nuclear radius, and $H_{\text {dip quad }}$ describes the coupling between the giant resonances and the quadrupole vibrations. Analogous to the Coulomb inter- 
action, the nuclear interaction is assumed to be

$$
H_{\text {Yukawa int }}=\frac{V_{Q}}{4 \pi} \int_{0}^{R_{1}\left(\theta_{1}, \theta_{1}\right)} d \tau_{1} \rho\left(\overrightarrow{\mathrm{r}}_{1}\right) \int_{0}^{R_{2}\left(\theta_{2}, \theta_{2}\right)} d \tau_{2} \rho\left(\overrightarrow{\mathrm{r}}_{2}\right) \frac{e^{-\left|\overrightarrow{\mathrm{r}}_{1}-\overrightarrow{\mathrm{r}}_{2}-\overrightarrow{\mathrm{R}}\right| / \mu}}{\mid \overrightarrow{\mathrm{r}}_{1}-\overrightarrow{\mathrm{r}}_{2}-\overrightarrow{\mathrm{R}}}
$$

with $\rho(\overrightarrow{\mathrm{r}})=A / V$. The integral is expanded up to first order in $\alpha_{20}$ and $\alpha_{30}$ in the upper boundary and solved by a Fourier transformation. Finally we evaluate the strength $V_{0}$ of the Yukawa force using the method of Scheid et al. for a homogenous density of nuclear matter. ${ }^{9,}{ }^{10}$. They made the following Ansatz for the dependence of the binding energy on the nuclear density:

$$
E(\rho)=W_{0} A+\frac{C}{2 \rho_{0}} \int\left(\rho-\rho_{0}\right)^{2} d \tau+\frac{3}{5} \frac{(Z e)^{2}}{R}+\frac{\kappa}{\rho_{0}}\left(2 \frac{Z}{A}-1\right)^{2} \rho V+E_{\text {Yukawa }}
$$

with

$$
E_{\text {Yukawa }}=-V_{0} \rho^{2} \frac{R_{0}^{2} \pi}{K^{3}}\left[1-\frac{1}{K^{2} R_{0}{ }^{2}}+e^{-2 K R_{0}\left(1+\frac{1}{K R_{0}}\right)^{2}}\right]
$$

and $K=1 / \mu$. The constant $W_{0}$ is the binding energy of a nucleon in infinite nuclear matter. The second term denotes the compression energy, while the following terms describe the Coulomb, symmetry, and Yukawa energies, respectively. The values for $W_{0}$ and the equilibrium density $\rho_{0}$ are taken from nuclear-matter theory: $W_{0}=15.85$ $\mathrm{MeV}, \rho_{0}=0.2 \mathrm{fm}^{-3}$. The binding energy should be stable with respect to $R_{0}$ and $Z$, i.e., for a given range $\mu$ the other three constants are determined by the binding energy and the conditions

$$
\partial E / \partial Z=0, \quad \partial E / \partial R_{0}=0 .
$$

$V_{0}, C$, and $\kappa$ are rather independent of the mass number $A$; $C$ has a value of about $14 \mathrm{MeV}, \kappa$ of about $30 \mathrm{MeV}$.

We have now established and solved the classical Hamilton equations for a given energy $E_{i}$ of the projectile starting at large distances. $E_{i}$ is chosen such that the two nuclear surfaces just come into contact, if the deformation of the sur-

Table I. Calculated fusion barriers $E_{i}=E_{\text {fusion }}$ as a function of the range of the nuclear force. The strengths of the Yukawa force as obtained for a given range $\mu$ [see Eq. (6)] are listed in the second column.

\begin{tabular}{ccc}
\hline $\begin{array}{c}\mu \\
(\mathrm{fm})\end{array}$ & $\begin{array}{c}V_{0} \\
(\mathrm{MeV} \mathrm{fm})\end{array}$ & $\begin{array}{c}E_{i} \\
(\mathrm{MeV})\end{array}$ \\
\hline \multicolumn{3}{c}{${ }^{148} \mathrm{Nd}-{ }^{148} \mathrm{Nd}$} \\
0.6 & -723 & 408 \\
0.8 & -307 & 401 \\
1.0 & -159 & 396 \\
1.2 & -93.1 & 392 \\
& $122 \mathrm{Sn}-{ }^{122} \mathrm{Sn}$ & \\
0.6 & -741 & 288 \\
0.8 & -315 & 282 \\
1.0 & -163 & 277 \\
1.2 & -95.8 & 273 \\
\hline
\end{tabular}

face during the collision is taken into account. The new fusion barrier is compared with the Coulomb barrier of two undeformed nuclei

$$
E_{\mathrm{C}}=Z_{1} Z_{2} e^{2} /\left(R_{0(1)}+R_{0(2)}\right) .
$$

Tables I and II show the results. It is striking that one obtains in every case $E_{i}<E_{\mathrm{C}}$, i.e., the Yukawa potential overcompensates the effect of the Coulomb potential. This becomes clear from the excitation of the internal degrees of freedom shown in Fig. 1. The giant resonances are of high frequency and thus follow the orbital path almost adiabatically. Therefore the amplitude $d_{10}$ of the giant resonances is nearly zero after the collision. In contrast to this, the octupoles $\left(\alpha_{30}\right)$ and the quadrupoles $\left(\alpha_{20}\right)$ oscillate with relatively large amplitudes. This can be seen from Figs. 1(b) and 1(c) which show the excitation energy of the various degrees of freedom as a function of time. At first the quadrupoles are strongly excited by the Coulomb force. The nuclear forces are for quadrupoles and octupoles of the same order of magnitude. Since the inertia parameter of the octupoles is much smaller than the inertia parameter of the quadrupoles

Table II. Fusion barriers in $\mathrm{MeV}$ for different nuclei $(\mu=0.8 \mathrm{fm})$. The numbers in the brackets show the Coulomb barrier $E_{C}$ [Eq. (8)] of two undeformed nuclei.

\begin{tabular}{cccc}
\hline & ${ }^{106} \mathrm{Pd}$ & ${ }^{122} \mathrm{Sn}$ & ${ }^{148} \mathrm{Nd}$ \\
\hline${ }^{148} \mathrm{Nd}$ & & & 401 \\
& & & $(408.9)$ \\
${ }^{122} \mathrm{Sn}$ & & 282 & 334 \\
& & $(302.8)$ & $(351.7)$ \\
${ }^{106} \mathrm{Pd}$ & 259 & 269 & 322 \\
& $(268.6)$ & $(285.1)$ & $(330.9)$ \\
\hline
\end{tabular}




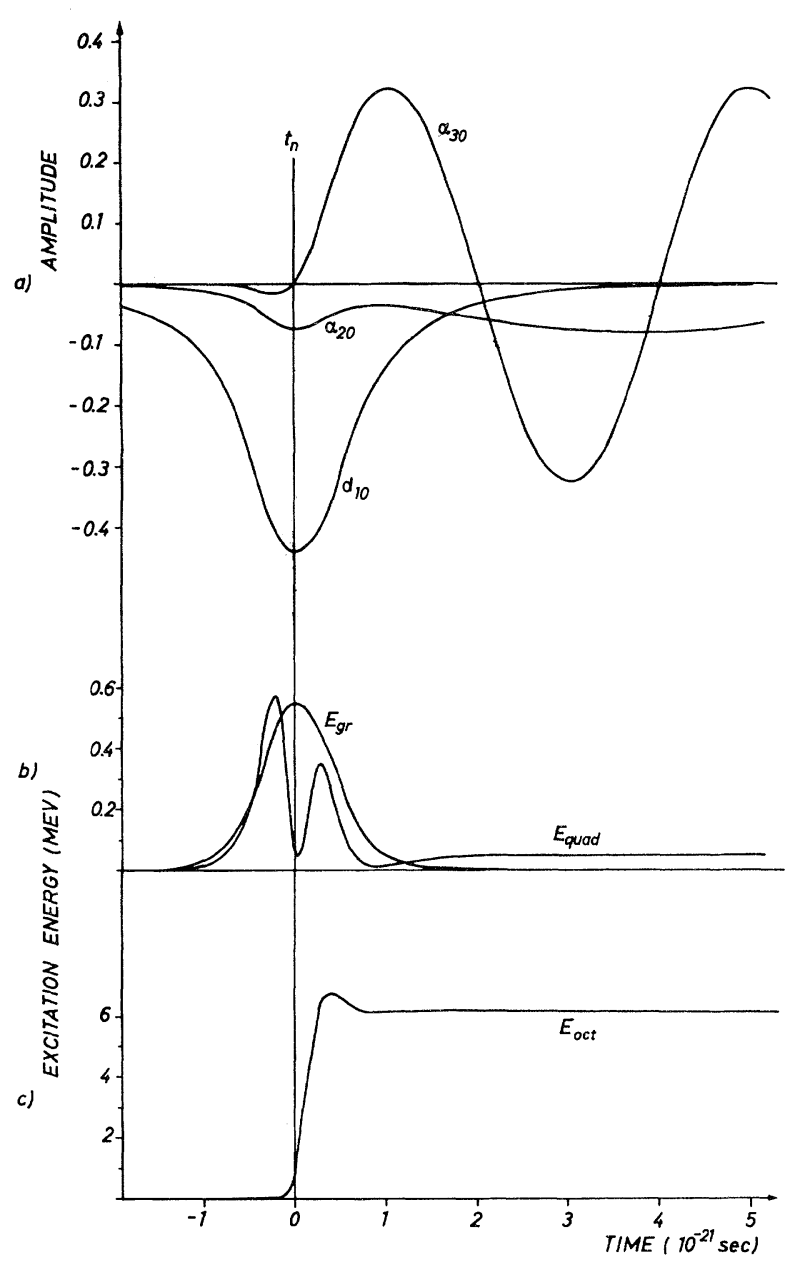

FIG. 1. Excitation of the collective modes in a ${ }^{148} \mathrm{Nd}-$ ${ }^{148} \mathrm{Nd}$ collision with the energy $E_{i}=401 \mathrm{MeV}$ and the nuclear parameters $V_{0}=-307 \mathrm{MeV} \mathrm{fm}$ and $\mu=0.8 \mathrm{fm}$. The point of nearest approach of the two centers of masses is marked by $t_{n}$. (a) Amplitudes of the quadrupole vibration $\alpha_{20}$, the octupole vibration $\alpha_{30}$, and the giant resonance $d_{10}$ as a function of time. (b) Excitation energies of the collective modes $E_{\text {quad }}$ and $E_{\text {gr }}$ as a function of time. (c) Excitation energy $E_{\text {oct }}$.

(and thus the oscillation frequency $\omega_{3}$ is larger than $\omega_{2}$ ), the octupole elongation can follow the nuclear force much faster than the quadrupole elongation [see Fig. 1(a)]. When the ions have separated again the strong nuclear forces have very much elongated the octupoles. The same is true, but to a lesser extent, for the quadrupoles. Since their frequency is smaller, the nuclear force is not able to elongate them as much as the octupoles during the collision time. As soon as the ions have separated so far that they are out of the range of the nuclear forces, the longrange Coulomb force still influences the quadrupoles and thus diminishes the effect of the Yukawa force. Consequently the final octupole excitation energy is much larger than the final quadrupole excitation energy. From this classical picture we see that at the Coulomb barrier only those modes are strongly excited for which $T_{n}$ $\leqslant T_{\text {osc }}$. Here $T_{n}$ is the typical time of action of the nuclear forces. For nuclei in the Nd region this condition is fulfilled for the octupole vibration, i.e., $T_{n} \approx \frac{1}{4} T_{\text {oct }}$. Since the Yukawa force favors a prolate shape of the nucleus the Coulomb barrier is considerably reduced, and the contact point of the two nuclear surfaces occurs always at a later time than the turning point $t_{n}$ of the disturbed Rutherford hyperbola.

We would like to thank Dr. W. Scheid for encouraging and stimulating discussions.

\footnotetext{
*Work supported by the Bundesministerium für Wissenschaftliche Forschung and the Deutsche Forschungsgemeinschaft.

${ }^{1}$ R. Beringer, Phys. Rev. Letters 18, 1006 (1967).

${ }^{2}$ H. Holm, W. Scheid, and W. Greiner, Phys. Letters 29B, 473 (1969).

${ }^{3}$ C. Y. Wong, Phys. Letters 26B, 120 (1968).

${ }^{4}$ H. Holm, D. Scharnweber, W. Scheid, and W. Greiner, to be published.

5. Eisenberg and W. Greiner, "Nuclear Theory" (to be published).

${ }^{6}$ M. Danos and W. Greiner, Phys. Rev. 134, B284 (1964).

${ }^{7}$ C. Y. Wong, Nucl. Data A4, 271 (1968).

${ }^{8}$ O. Hansen and O. Nathan, Nucl. Phys. 42, 197 (1963).

${ }^{9}$ W. Scheid, R. Ligensa, and W. Greiner, Phys. Rev. Letters 21, 1479 (1968).

${ }^{10}$ W. Scheid and W. Greiner, Z. Physik 226, 364 (1969).
} 